\title{
Alternative transitions between existing representations in multi-scale maps
}

\author{
Marion Dumont, ${ }^{\mathrm{a}}$ Guillaume Touya, ${ }^{\mathrm{a}}$ Cécile Duchêne ${ }^{\mathrm{a}}$ \\ ${ }^{a}$ Univ. Paris-Est, LASTIG COGIT, IGN, ENSG, F-94160 Saint-Mande,France; \{firstname.name\}@ign.fr
}

\begin{abstract}
Map users may have issues to achieve multi-scale navigation tasks, as cartographic objects may have various representations across scales. We assume that adding intermediate representations could be one way to reduce the differences between existing representations, and to ease the transitions across scales. We consider an existing multiscale map on the scale range from 1:25k to $1: 100 \mathrm{k}$ scales. Based on hypotheses about intermediate representations design, we build custom multi-scale maps with alternative transitions. We will conduct in a next future a user evaluation to compare the efficiency of these alternative maps for multi-scale navigation. This paper discusses the hypotheses and production process of these alternative maps.
\end{abstract}

Keywords: Multi-scale map, Intermediate Representation, Cartographic generalization, Smooth transitions

\section{Introduction}

A multi-scale map is a set of maps displayed at different scales, which can represent the geographic space with various levels of abstraction. Across scales, map users may have issues to recognize a same location across scales because of these changes of representations and can be disturbed while zooming. We assume that adding intermediate representations could be one way to reduce these differences and to ease the visual and cognitive transitions between existing maps.

To produce each intermediate representation, we face the cartographic choices of any map design process. Moreover, as these intermediate representations will be part of a multi-scale map, we have to make these choices considering the other existing maps. To get some guidelines, in a previous work (Dumont et al. 2016b) we studied the variation of these cartographic choices across scales in sixteen existing multi-scale maps, provided by National Mapping Agencies, private companies or collaborative communities. Based on this former study, we make assumptions about the variables of intermediate representations design, which could have an influence on multi-scale navigation.

To confirm or infirm our hypotheses, we will conduct a user evaluation in a near future, asking participants to achieve multi-scale navigation tasks on different multiscale maps, each one implementing a different hypothesis. This paper only discusses our first hypotheses and test material production.

The rest of the paper is structured as follows. Section 2 discusses our questions and hypotheses about transitions improvement. Section 3 presents our initial test material, from which we build alternative maps. Finally, Section 4 presents the modelling process of these alternative maps. Finally, Section 5 will conclude this paper.

\section{Hypotheses about transitions improvement}

This section introduces our hypotheses about intermediate representations design, in order to improve transitions in multi-scale maps. Then, for each hypothesis, a dedicated multi-scale map will be produced, as described in Section 4.

\subsection{Number of intermediate representations}

We wonder if the number of intermediate representations influences the multi-scale navigation and which number is relevant to improve the transitions between two specific existing representations. Comparing multi-scale maps having a different number of intermediate representations (1, 2 or 3 for instance) may show us the influence of this variable on the multi-scale navigation.

It is with noticing that we place our multi-scale maps in a continuous zooming environment (van Wijk and Nuij 2003), which enables very short scale gaps even if we only have few intermediate representations. When the user zooms in such system, the current representation is magnified, until the display scale of the next available representation is reached.

\subsection{Display scales for additional zoom levels}

As highlighted in Dumont et al. (2016b), some multiscale maps use a same map only magnified at several zoom levels. In these cases, intermediate representations could be used at these existing zoom levels, instead of the current magnified ones, to improve the content transition across scales. In other cases, to simplify transitions between two different maps, we should add zoom levels. Then the question is: how should we choose the display scales of these new zoom levels?

As most producers apply the WMTS standard (Dumont et al. 2016b), we propose to also use it for intermediate levels. Instead of its quad tree structure and the associated quadratic scale progression, we could also divide the 
studied scale range on equal scale steps. Besides, we could also use 'critical scales' inspired by Ratajski (1967), adding an intermediate representation as soon as an important change of representation is needed. However, the definition of these 'critical scales' could be very time-consuming as we should produce a lot of maps. Comparing multi-scale maps with the same content, but with different scales sets could give us insights about the influence of the scale evolution on the multi-scale navigation. However, this comparison will need an important scale range, to obtain sufficiently different multi-scale maps and measure something relevant.

\subsection{Transitions between levels of abstraction}

Depending on the map scale and purpose, the level of abstraction defines the semantic level of detail with which the geographic space is described (Mackaness 2007). The transitions between maps with a different level of abstraction could be very disturbing for the user, as the visual changes are quite important. This way, the Figure 1 shows four different levels of abstraction for settlement areas: individual buildings, urban blocks, urban areas and city point symbols.

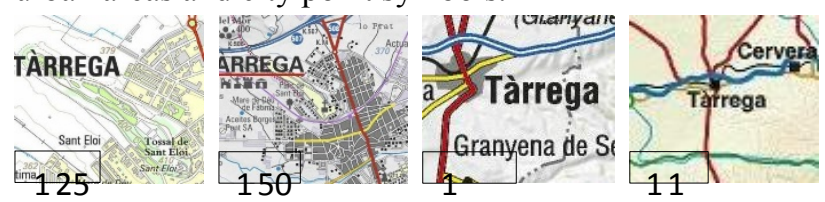

Fig. 1. A same geographic object may have very different representations across scales, as these settlement areas (Source: ICGC Catalonia)

In a previous study (Dumont et al. 2016b), we identified interesting patterns of 'mixed representations', which could help to smooth direct transitions. For instance, the Figure 2 shows alternatives to direct transitions between individual buildings and urban areas: a progressive aggregation into urban blocks or a superimposition of individual buildings and urban areas, which visually explicit the relationships between the two levels of abstraction. Comparing multi-scale maps implementing these different patterns could help us to get insight on the benefits brought by such mixed representations.

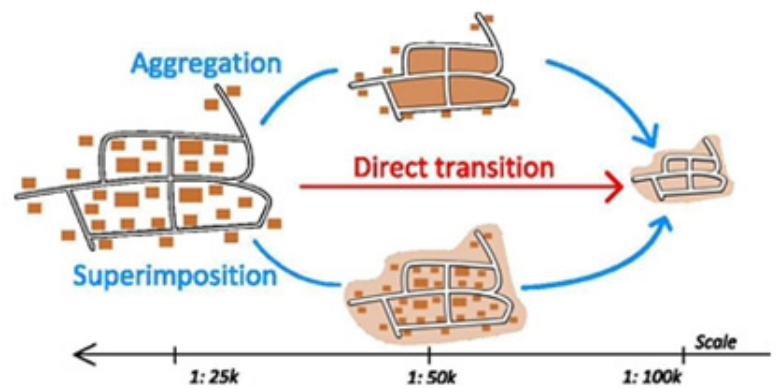

Fig. 2. The three different patterns of transitions between two different levels of abstraction of settlement areas: direct transition between individual buildings and urban areas, transition by progressive aggregation into urban blocks, transition by superimposition of urban areas under individua buildings (Source: Dumont et al. 2016b)

\subsection{Generalization Process}

Once the scale and the level of abstraction of the intermediate representation have been chosen, the choice of the generalization process still remains. To produce consistent intermediate representations, this process should take into account the existing representations at smaller scale (Girres and Touya 2014). Then when possible, continuous generalization operators (van Kreveld 2001) could be used to produce smooth geometric transitions.

We assume that preserving landmarks (Elias 2003) and spatial patterns (Mackaness and Edwards 2002) could help the user in his multi-scale navigation task. We thus want to test if keeping these objects in intermediate representations could improve the transitions.

We also want to test if relevant generalization processes for mono-scale map also apply to multi-scale maps, as for instance the agent-based generalization processes (Ruas and Plazanet 1996; Barrault et al. 2001; Duchęne et al. 2012), or if some inconsistencies appear when using it at several scales.

\section{Initial Material}

To produce our custom multi-scale map, we choose as initial situation the "Scan Express multi-scale map", produced by the French National Mapping Agency. This multi-scale map uses a similar style at all scales, which ensures a homogeneous symbolization. However, as the different maps have been derived from different databases, some inconsistencies appear. For instance, Figure 3 shows a salient highway which changes color across scales. We choose to correct these inconsistencies in existing maps, to avoid their influence on task performances during the test. After checking on the study area, the existing maps were found consistent in terms of content.

To limit the time of map making and the quantity of variables to test, we choose to only consider the scale range

between $1: 25 \mathrm{k}$ and $1: 100 \mathrm{k}$. Three cartographic datasets exist on this scale range, produced at 1: $25 \mathrm{k}, 1: 50 \mathrm{k}$ and 1: $100 \mathrm{k}$ scales. Extracts of these maps are presented in Figure 3. We only use the following geographic themes of topographic maps: land use, hydrography, roads, settlement areas and place names. This initial multi-scale map will be included in the user evaluation to serve as reference and confirm (or infirm) our hypotheses about transitions improvements.

\section{Production of Alternative Transitions}

This section describes the processes used to build each custom multi-scale map, called an "alternative" to the reference multi-scale map. To enable the comparison between alternatives and the reference multi-scale map, we use the existing datasets at $1: 25 \mathrm{k}$ and $1: 100 \mathrm{k}$ scales to produce them. However, to limit the constraints on intermediate representations design, we choose not to use the initial dataset at $1: 50 \mathrm{k}$ in the alternatives. Then in each alternative, we derive several intermediate representations, depending on the hypothesis to test.

We only discuss about the algorithms we use to produce alternative maps, so we do not discuss about the number and display scales of intermediate representations in this 
part. Besides, we particularly focus on the simplification of roads and settlement areas, as they are very salient cartographic themes. Due to generalization, these objects may have very different representations across scales.

\subsection{Transitions between roads representations}

Regarding roads, we identified the simplification of complex intersections as a key issue for smooth transitions. We want to test if adding a typified version of these complex intersections as an intermediate representation could improve the multi-scale navigation. The production of this alternative requires a process to detect and typify such complex intersections (Touya 2010). More globally, we also use a selection process from (Touya 2010) to generalize roads in intermediate representations. If needed, continuous roads simplification such as proposed in Nöllenburg et al. (2008) can also be helpful to produce smooth geometric changes between the initial $1: 25 \mathrm{k}$ geometry and the final $1: 100 \mathrm{k}$ geometry.

\subsection{Transitions \\ between settlement \\ areas representations}

\subsubsection{Mixed representations}

The representation of settlement areas may be even more heterogeneous across scales as shown in Figure 1. We want to test if mixed representations may improve a direct transition between maps with different levels of abstraction, as in our case study, between individual buildings at $1: 25 \mathrm{k}$ scale and urban areas at $1: 100 \mathrm{k}$ scale. We produce an alternative with a transition by aggregation of individual buildings into urban blocks. To build a progressive block covering across scales, we use an automatic process (Touya and Dumont 2017), based on buildings classification. Then, we also produce an alternative with a transition by superimposition of urban areas under all individual buildings. As remaining buildings in these two alternatives may need to be simplified, we also use a building typification process inspired by Burghardt and Cecconi (2007). Comparing the performances obtained with these three different alternatives may highlight the benefits and disadvantages of each pattern for smooth transitions across scales.

\subsubsection{Landmarks and spatial patterns}

We assume that preserving landmarks (Elias 2003) and spatial patterns (Mackaness and Edwards 2002) while generalizing may help the user to navigate across scales. To test the impact of landmarks, we build an alternative using the previous transition by aggregation, while keeping the buildings landmarks on top of urban blocks. To identify landmarks, we use an automatic process (Touya and Dumont 2017), adapted from (Elias 2003) with specific indicators for buildings (semantic nature, size, shape, localization etc.). Comparing these two alternatives with and without landmarks may highlight the efficiency of keeping landmarks for the multi-scale navigation.

To test the influence of spatial patterns, we build an alternative with a typification process inspired by Burghardt and Cecconi (2007), which preserves such spatial structures. In this case, we will compare this alternative with the initial situation or with another alternative using generalization processes that do not particularly preserve visual patterns. As the typification may not be sufficient to solve all the spatial conflicts at smaller scales, we cover urban blocks where building density is too high.

\subsubsection{Agent-based generalization processes}

Besides, we also build an alternative with an agent-based generalization processes previously used with success for static maps (Ruas and Plazanet 1996; Barrault et al. 2001; Touya and Duchęne 2011; Duchęne et al. 2012), to test if it obtains results with the same quality at different scales.

\subsection{Illustrations and Tables}

To individually evaluate each intermediate representation, we first can apply generalization evaluation methods for

static maps (Bard and Ruas 2005; Mackaness and Ruas 2007; Touya 2012). In addition, we should evaluate them regarding the whole multi-scale maps, measuring the variation of some indicators across scales, such as the visual complexity with clutter measures (Dumont et al. 2016a) or the amount of information with analytical measures (Harrie et al. 2015).

Then, to evaluate a whole alternative, we will conduct a user evaluation, asking participants to achieve a usual multiscale navigation task. Each participant will have to realize this task on the different alternatives. Then, comparing the tasks performances between alternatives, for instance the time and accuracy of tasks achievement, we will get insight about the benefits of each alternative for multi-scale navigation improvement.

\section{Captions}

\section{Conclusion}

In this paper, we discuss our hypotheses about intermediate representations design. Considering an existing multiscale map on the scale range from $1: 25 \mathrm{k}$ to $1: 100 \mathrm{k}$, we discuss the production of alternative transitions. In a next future, we will conduct a user evaluation, asking participants to achieve multi-scale task on these multi-scale maps. The comparison of performances between alternatives will give us insights about the benefits of each alternative for multiscale navigation improvement.

Building such intermediate representations raises more issues, considering the number of needed intermediate representations and their relative display scales, which could also be tested in a user evaluation. Besides, for each intermediate representation, the parameterization of generalization process should be studied, regarding the existing maps and in order to obtain progressive changes across scales.

Moreover, usual visualization systems for multi-scale maps are regularly based on tiled grid structured as a quad tree. Adding intermediate representations may require adaptations of these systems or even new solutions. 


\section{Acknowledgements}

This work is supported by the French National Research Agency, as part of the MapMuxing project [ANR-14CE24-0011-01].

\section{References}

Bard, S., Ruas, A. (2005). Why and How Evaluating Generalised Data?, in Developments in Spatial Data Handling, Springer Berlin Heidelberg, pp. 327-342

Barrault, M., Regnauld, N., Duchęne, C., Haire, K., Baeijs, C., Demazeau, Y., Hardy, P., Mackaness, W.A., Ruas, A., Weibel, R. (2001).

Integrating multi-agent, object-oriented, and algorithmic techniques for improved automated map generalisation. In Proceedings of 20th International Cartographic Conference (pp. 2110-2116). Beijing, China.

Burghardt, D., Cecconi, A. (2007). Mesh simplification for building typification. International Journal Geographic Information Science, 21(3), 283-298.

Duchęne, C., Ruas, A., Cambier, C. (2012). The CartACom model: transforming cartographic features into communicating agents for cartographic generalisation. International Journal of Geographical Information Science, 26(9), 1533-1562.

Dumont, M., Touya, G., Duchęne, C. (2016a). AGILE workshop on "Automated generalisation for on-demand mapping" and 19th ICA Workshop on "Generalisation and Multiple Representation", Helsinki, 2016

Dumont, M., Touya, G., Duchęne, C. (2016b). A Comparative Study of Existing Multi-Scale Maps: What Content at Which Scale? In: GIScience 2016. Montreal, Canada

Elias, B. (2003). Extracting landmarks with data mining methods. In Kuhn, W., Worboys, M. F., and Timpf, S. (Eds.), Spatial Information Theory. Foundations of Geographic Information Science (pp. 375-389). Berlin Heidelberg: Springer.

Girres, J.F., Touya, G. (2014). Cartographic Generalisation Aware of Multiple Representations, In Proceedings of GIScience 2014 - Poster session, (Eds) Duckham M, Stewart K, Pebesma E

Harrie, L., Stigmar, H., Djordjevic, M. (2015). Analytical Estimation of Map Readability. ISPRS International Journal of Geo-Information, 4, 418-446

Mackaness, W.A., Edwards, G. (2002). The Importance of Modelling Pattern and Structure in Automated Map Generalisation., in Proceedings of the Joint ISPRS/ICA Workshop on Multi-Scale Representations of Spatial Data (pp. 7-8)

Mackaness, W., Ruas, A. (2007). Evaluation in the Map generalisation Process, in Generalisation of geographic information: cartographic modelling and applications, S. T. Mackaness W. Ruas A., Ed. Elsevier, 2007

Nöllenburg, M., Merrick, D., Wolff, A., Benkert, M. (2008). Morphing polylines: A step towards continuous generalization, Computers, Environment and Urban Systems, 32 (4), 248-260
Ratajski, L. (1996). Phénomčnes des points de généralisation, International Yearbook of Cartography, 7, 143-152

Ruas, A., Plazanet, C. (1996). Strategies for automated generalization. In 7th International Symposium on Spatial Data Handling (pp. 319-336), Delft, Netherlands.

Touya, G. (2010). A Road Network Selection Process Based on Data Enrichment and Structure Detection, Transactions in GIS, 14(5), 595-614

Touya, G. (2012). Social welfare to assess the global legibility of a generalized map. In N. Xiao, M.P. Kwan, M.F. Goodchild, S. Shekhar, Eds. , Geographic Information Science 7th International Conference, GIScience 2012. Berlin, Heidelberg: Springer Berlin Heidelberg, pp. 198-211. Springer Berlin / Heidelberg, Berlin, Heidelberg.

Touya, G., Duchęne, C. (2011). CollaGen: Collaboration between automatic cartographic Generalisation Processes," in Advances in Cartography and GIScience, vol. 1, A. Ruas, Ed. Berlin, Heidelberg: Springer Berlin Heidelberg, pp. 541-558

Touya, G., Dumont, M. (2017). Progressive Block Graying and Landmarks Enhancing as Intermediate Representations between Buildings and Urban Areas. In Proceedings of 20th ICA Workshop on Generalisation and Multiple Representation, Washington D.C, submitted

Van Kreveld M (2001) Smooth generalization for continuous zooming, Proceedings of 20th International Cartographic Conference, ICA, Beijing, China, pp. 2180-2185

Van Wijk, J. J., Nuij, W. A. (2003).“Smooth and efficient zooming and panning," in Information Visualization, 2003. INFOVIS 2003. IEEE Symposium on, 2003, pp. $15-23$ 